\title{
UMA CLASSE DE PROBLEMAS DE CONTROLE ÓTIMO EM ESCALAS TEMPORAIS
}

\author{
Iguer L.D. Santos*, Geraldo N. Silva ${ }^{\dagger}$ \\ ${ }^{*}$ Rua Rio de Janeiro, 266 \\ UNESP - Universidade Estadual Paulista \\ Ilha Solteira, SP, Brasil \\ ${ }^{\dagger}$ Rua Cristóvão Colombo, 2265 \\ UNESP - Universidade Estadual Paulista \\ São José do Rio Preto, SP, Brasil
}

Emails: iguerluis@yahoo.com.br, gsilva@ibilce.unesp.br

\begin{abstract}
In this paper we consider a class of optimal control problems on time scales. Using a extension of Filippov's Lemma to control systems in time scales and a result of compactness of trajectories for dynamic inclusions on time scales, we present conditions for the existence of solutions to this class of optimal control problems.

Keywords - Optimal control, Existence, Time scales, Filippov's measurable selection.

Resumo - Nesse artigo consideramos uma classe de problemas de controle ótimo em escalas temporais. Usando uma extensão do Lema de Filippov para sistemas de controle em escalas temporais e um resultado de compacidade das trajetórias para inclusões dinâmicas em escalas temporais, apresentamos condições para a existência de soluções para essa classe de problemas de controle ótimo.
\end{abstract}

Palavras-chave- Controle ótimo, Existência, Escalas temporais, Seleção mensurável de Filippov.

\section{Introdução}

O cálculo em escalas temporais foi introduzido por Hilger (Hilger, 1990) para unificar o cálculo de diferença e o cálculo diferencial. A teoria de escalas temporais admite diversas aplicações em modelagem matemática e outras áreas, conforme verificamos em (Agarwal et al., 2002), (Bohner and Peterson, 2003), (Bohner et al., 2011), (Bohner and Guseinov, 2010), (Federson et al., 2012), (Santos and Silva, 2013) e (Zhan et al., 2009). Em particular, problemas de controle ótimo em escalas temporais foram considerados, por exemplo, em (Gong and Xiang, 2009), (Hilscher and Zeidan, 2011), (Liu et al., 2011), (Peng et al., 2011) e (Zhan and Wei, 2009).

Nesse artigo provamos a existência de soluções para uma classe de problemas de controle ótimo de sistemas governados por sistemas dinâmicos em escalas temporais. O resultado de existência é obtido usando um resultado de compacidade das trajetórias (Santos, 2011) e uma extensão do Lema de Filippov para sistemas de controle em escalas temporais.

O artigo está organizado como segue. Na próxima seção consideramos conceitos e resultados básicos da teoria de escalas temporais. A Seçao 3 apresenta propriedades de mensurabilidade de multifunções e a compacidade das trajetórias para inclusões dinâmicas. A extensão do teorema de seleção de Filippov para escalas temporais é provada na Seção 4. Finalmente, provamos a existência de processos ótimos para problemas de controle ótimo na Seção 5 e fazemos um breve comentário sobre este artigo na Seção 6.

\section{Preliminares}

Nesta seção consideramos conceitos e resultados básicos da teoria de escalas temporais que serão úteis no desenvolvimento desse artigo.

Utilizaremos as seguintes convenções:

i) se $x \in \mathbb{R}^{n}$ denotamos a norma euclidiana de $x$ por $\|x\|$.

ii) se $A$ e $\mathbb{T}$ são subconjuntos de $\mathbb{R}$, denotamos por $A_{\mathbb{T}}$ o conjunto $A \cap \mathbb{T}$.

iii) $B$ denotará o conjunto $\left\{x \in \mathbb{R}^{n}:\|x\| \leq 1\right\}$.

\subsection{Escala temporal}

Uma escala temporal $\mathbb{T} \subset \mathbb{R}$ é um conjunto nãovazio e fechado. Usaremos uma escala temporal $\mathbb{T}$ compacta, sendo $a=\min \mathbb{T}$ e $b=\max \mathbb{T}$.

Define-se a função $\sigma: \mathbb{T} \rightarrow \mathbb{T}$ como

$$
\sigma(t)=\inf \{s \in \mathbb{T}: s>t\}
$$

Estamos supondo que $\inf \emptyset=\sup \mathbb{T}$.

Lema 1 ((Cabada and Vivero, 2006))

Existem $I \subset \mathbb{N} e\left\{t_{i}\right\}_{i \in I} \subset \mathbb{T}$ tal que

$$
R S:=\{t \in \mathbb{T}: t<\sigma(t)\}=\left\{t_{i}\right\}_{i \in I} .
$$




\subsection{Integração em escalas temporais}

A $\sigma$-álgebra de subconjuntos da escala temporal $\mathbb{T}$, constituída de conjuntos $\Delta$-mensuráveis, pode ser encontrada em (Guseinov, 2003). Denotaremos essa $\sigma$-álgebra por $\Delta$ e a $\Delta$-medida de Lebesgue por $\mu_{\Delta}$.

Lema 2 ((Cabada and Vivero, 2006)) Tome $E \subset \mathbb{T}$. Então $E$ é $\Delta$-mensurável se, e somente se, $E$ é Lebesgue mensurável.

Para funções $f: \mathbb{T} \rightarrow \overline{\mathbb{R}}$ a noção de integração pode ser encontrada, por exemplo, em (Bartle, 1995), (Royden, 1988) e (Rudin, 1987). Denotamos a integral de uma função $f: \mathbb{T} \rightarrow \overline{\mathbb{R}}$ sobre um conjunto $E \in \Delta$ por

$$
\int_{E} f(s) \Delta s .
$$

Chamamos essa integral de $\Delta$-integral de Lebesgue de $f$ sobre $E$. Denotaremos por $L_{1}\left(E, \mathbb{R}^{n}\right)$ o conjunto das funções $f: \mathbb{T} \rightarrow \mathbb{R}^{n} \Delta$-integráveis sobre $E$.

Dada uma função $f: \mathbb{T} \rightarrow \mathbb{R}^{n}$ defina $\tilde{f}:$ $[a, b] \rightarrow \mathbb{R}^{n}$ por

$\tilde{f}(t)=\left\{\begin{array}{l}f(t), t \in \mathbb{T} \\ f\left(t_{i}\right), \quad t \in\left(t_{i}, \sigma\left(t_{i}\right)\right) \text { para algum } i \in I,\end{array}\right.$

onde $I \subset \mathbb{N}$ e $\left\{t_{i}\right\}_{i \in I}=R S$. De (Cabada and Vivero, 2006) sabe-se que a função $f$ é $\Delta$-mensurável se, e somente se, $\tilde{f}$ é Lebesgue mensurável.

Seja $E \subset \mathbb{T}$ e defina

$$
\tilde{E}=E \cup \bigcup_{i \in I_{E}}\left(t_{i}, \sigma\left(t_{i}\right)\right)
$$

onde

$$
I_{E}:=\left\{i \in I: t_{i} \in E \cap R S\right\} .
$$

O teorema a seguir é provado em (Cabada and Vivero, 2006) para funções escalares. Entretanto, esse resultado continua válido para funções vetoriais, como afirmado a seguir.

\section{Teorema 3 ((Cabada and Vivero, 2006))}

Seja $E \in \Delta$ tal que $b \notin E$. Então, $f \in L_{1}\left(E, \mathbb{R}^{n}\right)$ se, e somente se, $\tilde{f} \in L_{1}\left(\tilde{E}, \mathbb{R}^{n}\right)$. Neste caso,

$$
\int_{E} f(s) \Delta s=\int_{\tilde{E}} \tilde{f}(s) d s .
$$

Lema 4 ((Santos, 2011)) Seja $u: \mathbb{T} \rightarrow \mathbb{R}^{m}$ uma função $\Delta$-mensurável. Se A é Borel mensurável então $u^{-1}(A) \in \Delta$.

\section{Mensurabilidade de multifunções e compacidade de trajetórias}

Aqui apresentamos propriedades de multifunções mensuráveis e o resultado de compacidade de trajetórias. Estas propriedades serão usadas na obtenção de soluções para problemas de controle ótimo em escalas temporais.

\subsection{Multifunções mensuráveis}

Seja $(\Omega, \mathcal{F})$ um espaço mensurável. Uma multifunção $\Gamma: \Omega \rightsquigarrow \mathbb{R}^{n}$ é $\mathcal{F}$-mensurável quando o conjunto

$$
\Gamma^{-1}(V)=\{x \in \Omega: \Gamma(x) \cap V \neq \emptyset\}
$$

é $\mathcal{F}$-mensurável para cada conjunto compacto $V \subset$ $\mathbb{R}^{n}$.

Diz-se que uma multifunção $\Gamma$ é fechada, compacta, convexa ou não-vazia quando $\Gamma(x)$ satisfaz a propriedade exigida para cada $x \in \Omega$.

Teorema 5 ((Castaing and Valadier, 1977)) Tome um espaço mensurável $(\Omega, \mathcal{F})$ e uma multifunção $\Gamma: \Omega \rightsquigarrow \mathbb{R}^{m}$ não-vazia e fechada. Se $\Gamma$ é $\mathcal{F}$-mensurável então $\Gamma$ admite uma seleção mensurável.

Teorema 6 ((Castaing and Valadier, 1977)) Seja $\Gamma:[a, b] \rightsquigarrow \mathbb{R}^{m}$ uma multifunção fechada $e$ $E:=\{t \in[a, b]: \Gamma(t) \neq \emptyset\}$. Então as seguintes afirmações são equivalentes:

(a) a multifunção $\Gamma$ é $\mathcal{L}$-mensurável.

(b) o conjunto $\operatorname{Gr} \Gamma=\{(t, v): v \in \Gamma(t)\}$ é $\mathcal{L} \times$ $\mathcal{B}^{m}$-mensurável.

(c) o conjunto E é $\mathcal{L}$-mensurável e existe uma sequência de funções $\mathcal{L}$-mensuráveis $\gamma_{i}: E \rightarrow$ $\mathbb{R}^{m}$ tal que

$$
\Gamma(t)=\overline{\left\{\gamma_{i}(t): i=1,2, \ldots\right\}}
$$

para cada $t \in E$.

Corolário 7 Sejam $\Gamma_{1}, \Gamma_{2}:[a, b] \rightsquigarrow \mathbb{R}^{m}$ multifunções fechadas. Se $\Gamma_{1}$ e $\Gamma_{2}$ são $\mathcal{L}$-mensuráveis então a multifunção $\Gamma:[a, b] \rightsquigarrow \mathbb{R}^{m}$ dada por

$$
\Gamma(t)=\Gamma_{1}(t) \cap \Gamma_{2}(t)
$$

é $\mathcal{L}$-mensurável.

\subsection{Compacidade de trajetórias para inclusões dinâmicas}

Denotaremos por $A C\left(\mathbb{T}, \mathbb{R}^{n}\right)$ o conjunto de todas as funções absolutamente contínuas com domínio $\mathbb{T}$ e contradomínio $\mathbb{R}^{n}$. Também chamadas de arcos (Cabada and Vivero, 2005).

Considere uma multifunção $E: \mathbb{T} \times \mathbb{R}^{n} \rightsquigarrow$ $\mathbb{R}^{n}$ não-vazia. Dizemos que uma função $x \in$ $A C\left(\mathbb{T}, \mathbb{R}^{n}\right)$ é uma trajetória de $E$ se satisfizer a seguinte restrição

$$
x^{\Delta}(t) \in E(t, x(t)) \quad \Delta-q . t . p . \quad t \in[a, b)_{\mathbb{T}} .
$$

Teorema 8 ((Santos, 2011)) Seja $E: \mathbb{T} \times$ $\mathbb{R}^{n} \rightsquigarrow \mathbb{R}^{n}$ uma multifunção não-vazia, compacta, 
convexa e $\Delta \times \mathcal{B}^{n}$-mensurável. Considere uma função $c: \mathbb{T} \rightarrow[0,+\infty)$ em $L_{1}\left([a, b)_{\mathbb{T}}\right)$ tal que

$$
E(t, x) \subset(\gamma\|x\|+c(t)) B
$$

para todo $(t, x) \in \mathbb{T} \times \mathbb{R}^{n}$, sendo $\gamma>0$. Suponha que $\Delta$ - q.t.p. $t \in[a, b)_{\mathbb{T}}$ a multifunção $E(t,$.$) :$ $\mathbb{R}^{n} \rightsquigarrow \mathbb{R}^{n}$ possui o gráfico fechado.

Tome uma sequência de arcos $x_{i}: \mathbb{T} \rightarrow \mathbb{R}^{n}$ tal que $\left\{x_{i}(a)\right\}$ é limitada. Considere também uma sequência de funções $\Delta$-mensuráveis $y_{i}: \mathbb{T} \rightarrow \mathbb{R}^{n}$ tal que $y_{i}(t) \rightarrow 0 \Delta$-q.t.p. $t \in[a, b)_{\mathbb{T}}$. Suponha que existe uma função $\varphi: \mathbb{T} \rightarrow[0,+\infty)$ em $L_{1}\left([a, b)_{\mathbb{T}}\right)$ de modo que

$$
\left\|y_{i}(t)\right\| \leq \varphi(t)
$$

para todo $t \in \mathbb{T}$ e todo $i$.

Se para cada $i$ temos

$x_{i}^{\Delta}(t) \in E\left(t, x_{i}(t)+y_{i}(t)\right) \quad \Delta-q . t . p . \quad t \in[a, b)_{\mathbb{T}}$

então existe uma subsequência $\left\{x_{i_{k}}\right\} \subset\left\{x_{i}\right\} e$ uma trajetória $x$ de $E$ tal que $x_{i_{k}} \rightrightarrows x$.

\section{Teorema de seleção de Filippov em escalas temporais}

No Teorema 12, obtemos uma extensão para escalas temporais do clássico Teorema de seleção de Filippov (Filippov, 1988). Outras extensões desse teorema de seleção para escalas temporais foram obtidas em (Pawłuszewicz and Torres, 2010) e (Santos, 2011).

Considere o sistema de controle de equações

$$
\left\{\begin{array}{l}
x^{\Delta}(t)=f(\sigma(t), x(t), u(t)) \Delta-\text { q.t.p. } t \in[a, b)_{\mathbb{T}} \\
u(t) \in U(t) \Delta-\text { q.t.p. } t \in[a, b)_{\mathbb{T}} .
\end{array}\right.
$$

Se um par de processo de controle $(x, u)$ satisfaz a restrição 1 então $x$ satisfaz a seguinte inclusão dinâmica (2)

$$
x^{\Delta}(t) \in F(t, x(t)) \Delta-\text { q.t.p. } t \in[a, b)_{\mathbb{T}}
$$

sendo $F(t, x)=\{f(\sigma(t), x, u): u \in U(t)\}$. No Teorema 12 provaremos a afirmação inversa. Isto é, se um arco $x \in A C\left([a, b]_{\mathbb{T}}, \mathbb{R}^{n}\right)$ satisfaz (2) então existe uma seleção $\Delta$-mensurável $u$ de $U$ tal que o processo $(x, u)$ satisfaz $(1)$.

Tome uma função $\phi: \mathbb{T} \times \mathbb{R}^{m} \rightarrow \mathbb{R}^{n}$. Dizemos que $\phi$ é uma $\Delta$-função de Carathéodory se satisfizer as seguintes propriedades:

(a) para cada $t \in \mathbb{T}$ a função $x \mapsto \phi(t, x)$ é contínua.

(b) para cada $x \in \mathbb{R}^{m}$ a função $t \mapsto \phi(t, x)$ é $\Delta$-mensurável.

Lema 9 ((Loewen, 1993)) Seja $\phi:[a, b] \times$ $\mathbb{R}^{m} \rightarrow \mathbb{R}^{n}$ uma $\mathcal{L}$-função de Carathéodory. Então $\phi$ é $\mathcal{L} \times \mathcal{B}^{m}$-mensurável.
Lema 10 ((Santos, 2011)) Seja $\phi: \mathbb{T} \times \mathbb{R}^{m} \rightarrow$ $\mathbb{R}^{n}$ uma $\Delta$-função de Carathéodory. Então a função $\tilde{\phi}:[a, b] \times \mathbb{R}^{m} \rightarrow \mathbb{R}^{n}$ definida como

$$
\tilde{\phi}(t, u)=\left\{\begin{array}{l}
\phi(t, u), t \in \mathbb{T} \\
\phi\left(t_{i}, u\right), t \in\left(t_{i}, \sigma\left(t_{i}\right)\right) \text { para } i \in I .
\end{array}\right.
$$

é uma $\mathcal{L}$-função de Carathéodory.

Lema 11 ((Santos, 2011)) Seja $U: \mathbb{T} \rightsquigarrow \mathbb{R}^{m}$ uma multifunção $\Delta$-mensurável. Então a multifunção $\tilde{U}:[a, b] \rightsquigarrow \mathbb{R}^{m}$ definida como

$$
\tilde{U}(t)=\left\{\begin{array}{l}
U(t), \quad t \in \mathbb{T} \\
U\left(t_{i}\right), \quad t \in\left(t_{i}, \sigma\left(t_{i}\right)\right) \text { para } i \in I .
\end{array}\right.
$$

é $\mathcal{L}$-mensurável.

O Teorema a seguir é provado nas mesmas linhas do resultado de seleção obtido em (Santos, 2011).

Teorema 12 Seja $U: \mathbb{T} \rightsquigarrow \mathbb{R}^{m}$ uma multifunção não-vazia, fechada e $\Delta$-mensurável. Considere uma função $f: \mathbb{T} \times \mathbb{R}^{n} \times \mathbb{R}^{m} \rightarrow \mathbb{R}^{n}$ contínua em $(x, u)$ para cada $t$ fixado, e Borel mensurável em t para cada $(x, u)$ fixado.

Se $x \in A C\left([a, b]_{\mathbb{T}}, \mathbb{R}^{n}\right)$ satisfaz (2) então existe uma seleção $\Delta$-mensurável $u$ de $U$ tal que o processo $(x, u)$ satisfaz (1).

Prova: Seja $u \in \mathbb{R}^{m}$ fixado arbitrariamente. Como a função $\sigma$ é $\Delta$-mensurável (Guseinov, 2003), do Lema 4 a função $\varphi: \mathbb{T} \times \mathbb{R}^{n} \rightarrow \mathbb{R}^{n}$ dada por

$$
\varphi(t, x)=f(\sigma(t), x, u)
$$

é uma $\Delta$-função de Carathéodory.

Do Lema 10 a função $\tilde{\varphi}:[a, b] \times \mathbb{R}^{n} \rightarrow \mathbb{R}^{n}$ é uma $\mathcal{L}$-função de Carathéodory. Assim, do Lema 9 a função $\tilde{\varphi}$ é $\mathcal{L} \times \mathcal{B}^{n}$-mensurável.

Sendo $x$ uma função $\Delta$-mensurável, a função $\tilde{x}:[a, b] \rightarrow \mathbb{R}^{n}$ é $\mathcal{L}$-mensurável. Logo, a função

$$
t \mapsto \tilde{\varphi}(t, \tilde{x}(t))
$$

é $\mathcal{L}$-mensurável.

Defina a função $g: \mathbb{T} \rightarrow \mathbb{R}^{n}$ como

$$
g(t)=f(\sigma(t), x(t), u)
$$

Sendo $\tilde{\varphi}(t, \tilde{x}(t))=f(\sigma(t), x(t), u)$ para $t \in \mathbb{T}$ e $\tilde{\varphi}(t, \tilde{x}(t))=f\left(\sigma\left(t_{i}\right), x\left(t_{i}\right), u\right)$ para $t \in\left(t_{i}, \sigma\left(t_{i}\right)\right)$ para algum $i \in I$, temos que $\tilde{\varphi}(t, \tilde{x}(t))=\tilde{g}(t)$. Assim, a função

$$
t \mapsto \tilde{g}(t)
$$

\section{é $\mathcal{L}$-mensurável.}

Seja $m:[a, b] \times \mathbb{R}^{m} \rightarrow \mathbb{R}^{n}$ a função definida como $m(t, u)=f(\sigma(t), x(t), u)$ se $t \in \mathbb{T}$ e $m(t, u)=f\left(\sigma\left(t_{i}\right), x\left(t_{i}\right), u\right)$ se $t \in\left(t_{i}, \sigma\left(t_{i}\right)\right)$ para algum $i \in I$.

Para cada $u \in \mathbb{R}^{m}$ já provamos que a função

$$
t \mapsto m(t, u)
$$


é $\mathcal{L}$-mensurável. Além disso, se $t \in \mathbb{T}$ então a função

$$
u \mapsto m(t, u)=f(\sigma(t), x(t), u)
$$

é contínua. Por outro lado, se $t \in[a, b] \backslash \mathbb{T}$ seja $i \in I$ tal que $t \in\left(t_{i}, \sigma\left(t_{i}\right)\right)$, logo a função

$$
u \mapsto m(t, u)=f\left(\sigma\left(t_{i}\right), x\left(t_{i}\right), u\right)
$$

é contínua.

Como $x$ é um arco, a função $h: \mathbb{T} \rightarrow \mathbb{R}^{n}$ definida como

$$
h(s)=x^{\Delta}(s)
$$

é $\Delta$-mensurável. Logo, a função $\tilde{h}:[a, b] \rightarrow \mathbb{R}^{n}$ é $\mathcal{L}$-mensurável. Assim, a função $\phi:[a, b] \times \mathbb{R}^{m} \rightarrow$ $\mathbb{R}^{n}$ dada por

$$
\phi(t, u)=m(t, u)-\tilde{h}(t)
$$

é uma $\mathcal{L}$-função de Carathéodory.

Defina a multifunção $\Phi:[a, b] \rightsquigarrow \mathbb{R}^{m}$ como

$$
\Phi(t)=\left\{u \in \mathbb{R}^{m}: \phi(t, u)=0\right\}
$$

para cada $t \in[a, b]$.

Para cada $t \in[a, b]$ a função

$$
u \mapsto \phi(t, u)
$$

é contínua. Assim, se $\Phi(t) \neq \emptyset$ então $\Phi(t)$ é um conjunto fechado.

Do Lema 9 temos que $\phi$ é $\mathcal{L} \times \mathcal{B}^{m}$-mensurável. Logo, o conjunto

$$
\begin{aligned}
G r \Phi & =\left\{(t, u) \in[a, b] \times \mathbb{R}^{m}: u \in \Phi(t)\right\} \\
& =\left\{(t, u) \in[a, b] \times \mathbb{R}^{m}: \phi(t, u)=0\right\} \\
& =\phi^{-1}(\{0\})
\end{aligned}
$$

é $\mathcal{L} \times \mathcal{B}^{m}$-mensurável. Do Teorema 6 a multifunção $\Phi$ é $\mathcal{L}$-mensurável.

Como a multifunção $\tilde{U}:[a, b] \rightsquigarrow \mathbb{R}^{m}$ é $\mathcal{L}$ mensurável, do Corolário 7 a multifunção $\Gamma$ : $[a, b] \rightsquigarrow \mathbb{R}^{m}$ dada por

$$
\Gamma(t)=\tilde{U}(t) \cap \Phi(t)
$$

é $\mathcal{L}$-mensurável.

Considere os seguintes conjuntos

$$
\mathcal{K}=\left\{t \in[a, b)_{\mathbb{T}}:(2) \text { vale }\right\}
$$

e

$$
\mathcal{W}=\left\{t \in[a, b)_{\mathbb{T}}: U(t) \cap \Phi(t) \neq \emptyset\right\} .
$$

Então $\mathcal{W}=\mathcal{K}$ e o conjunto $E$ dado por

$$
E=\left\{t \in[a, b)_{\mathbb{T}}: U(t) \cap \Phi(t)=\emptyset\right\}
$$

satisfaz

$$
E=[a, b)_{\mathbb{T}} \backslash \mathcal{W}=[a, b)_{\mathbb{T}} \backslash \mathcal{K}
$$

Portanto

$$
\mu_{\Delta}(E)=\mu_{\Delta}\left([a, b)_{\mathbb{T}} \backslash \mathcal{K}\right)=0 .
$$

Se definirmos a multifunção $\Lambda: \mathbb{T} \rightsquigarrow \mathbb{R}^{m}$ como

$\Lambda(t)=\left\{\begin{array}{l}U(t), t \in E \cup\{b\} \\ U(t) \cap \Phi(t)=\Gamma(t), t \in \mathbb{T} \backslash\{E \cup\{b\}\}\end{array}\right.$

então $\Lambda$ é não-vazia e fechada.

Como $U$ é $\Delta$-mensurável e $\Gamma$ é $\mathcal{L}$-mensurável, usando o Lema 2 concluímos que $\Lambda$ é $\Delta$ mensurável.

Do Teorema 5 a multifunção $\Lambda$ admite uma seleção $\Delta$-mensurável $u: \mathbb{T} \rightarrow \mathbb{R}^{m}$. Logo, $u(t) \in$ $U(t)$ para todo $t \in \mathbb{T}$ e então $u$ também é uma seleção de $U$.

Se $t \in[a, b)_{\mathbb{T}} \backslash E$ temos que $u(t) \in U(t) \cap \Phi(t)$. Assim,

$$
\begin{aligned}
0 & =\phi(t, u(t))=m(t, u(t))-\tilde{h}(t) \\
& =f(\sigma(t), x(t), u(t))-h(t) \\
& =f(\sigma(t), x(t), u(t))-x^{\Delta}(t)
\end{aligned}
$$

isto é,

$$
x^{\Delta}(t)=f(\sigma(t), x(t), u(t)) .
$$

Portanto

$$
x^{\Delta}(t)=f(\sigma(t), x(t), u(t)) \Delta-q . t . p . t \in[a, b)_{\mathbb{T}}
$$

já que $\mu_{\Delta}(E)=0$. Então $(x, u)$ satisfaz a restrição dinâmica (1).

\section{Problemas de controle ótimo em escalas temporais}

Ao melhor de nosso conhecimento, não existe muita literatura discutindo a questão de existência de processos ótimos para problemas de controle ótimo em escalas temporais quando o estado é vetorial. Assim, procedendo como no caso contínuo (Vinter, 2000), usamos o teorema de seleção de Filippov para obter a existência de soluções para problemas de controle ótimo em escalas temporais.

Seja $g: \mathbb{R}^{n} \times \mathbb{R}^{n} \rightarrow \mathbb{R}$ uma função semicontínua inferior e $F: \mathbb{T} \times \mathbb{R}^{n} \rightsquigarrow \mathbb{R}^{n}$ uma multifunção. Considere o problema $(\mathrm{P})$ de controle ótimo

$$
\left\{\begin{array}{l}
\min g(x(a), x(b)) \text { sobre } x \in A C\left(\mathbb{T}, \mathbb{R}^{n}\right) \\
x^{\Delta}(t) \in F(t, x(t)) \Delta-\text { q.t.p. } t \in[a, b)_{\mathbb{T}} \\
(x(a), x(b)) \in A \times C
\end{array}\right.
$$

sendo $A, C \subset \mathbb{R}^{n}$.

Dizemos que $x \in A C\left([a, b]_{\mathbb{T}}, \mathbb{R}^{n}\right)$ é uma trajetória admissível do problema $(P)$, se $x$ é uma trajetória de $F$ que satisfaz as condições de fronteira $(x(a), x(b)) \in A \times C$.

Uma função $\bar{x} \in A C\left([a, b]_{\mathbb{T}}, \mathbb{R}^{n}\right)$ é uma trajetória ótima do problema $(P)$, se toda trajetória admissível $x$ de $(P)$ satisfizer

$$
g(\bar{x}(a), \bar{x}(b)) \leq g(x(a), x(b)) .
$$


Teorema 13 ((Santos, 2011)) Seja F uma multifunção não-vazia, compacta, convexa e $\Delta \times \mathcal{B}^{n}$-mensurável. Considere uma função $c: \mathbb{T} \rightarrow[0,+\infty)$ em $L_{1}\left([a, b)_{\mathbb{T}}\right)$ tal que

$$
F(t, x) \subset(\gamma\|x\|+c(t)) B
$$

para todo $(t, x) \in \mathbb{T} \times \mathbb{R}^{n}$, sendo $\gamma>0$. Suponha que $\Delta-$ q.t.p. $t \in[a, b)_{\mathbb{T}}$ a multifunção $F(t,$.$) :$ $\mathbb{R}^{n} \rightsquigarrow \mathbb{R}^{n}$ possui o gráfico fechado.

Suponha que $A$ é um conjunto compacto e $C$ é um conjunto fechado. Se o problema $(P)$ possui uma trajetória admissivel então existe uma trajetória ótima.

Prova: A prova é uma fácil consequência do Teorema 8 .

Considere agora uma função $f: \mathbb{T} \times \mathbb{R}^{n} \times$ $\mathbb{R}^{m} \rightarrow \mathbb{R}^{n}$ e uma multifunção $U: \mathbb{T} \rightsquigarrow \mathbb{R}^{m}$ nãovazia, compacta e $\Delta$-mensurável. Pode-se então considerar o seguinte problema (Q) de controle ótimo

$$
\left\{\begin{array}{l}
\min g(x(a), x(b)) \text { sobre }(x, u) \\
x^{\Delta}(t)=f(\sigma(t), x(t), u(t)) \Delta-\text { q.t.p. } \\
u(t) \in U(t) \Delta-\text { q.t.p. } t \in[a, b)_{\mathbb{T}} \\
(x(a), x(b)) \in A \times C
\end{array}\right.
$$

sendo $x \in A C\left([a, b]_{\mathbb{T}}, \mathbb{R}^{n}\right)$ e $u: \mathbb{T} \rightarrow \mathbb{R}^{m}$ uma função $\Delta$-mensurável.

Teorema 14 Suponha que a função $f: \mathbb{T} \times \mathbb{R}^{n} \times$ $\mathbb{R}^{m} \rightarrow \mathbb{R}^{n}$ satisfaça as seguintes condições:

(i) $f$ é contínua em $(x, u)$ para cada $t$ fixado, $e$ contínua em t para cada $(x, u)$ fixado.

(ii) o conjunto $f(\sigma(t), x, U(t))$ é convexo para cada $t \in \mathbb{T}$ e $x \in \mathbb{R}^{n}$.

(iii) existem $\gamma>0$ e c $>0$ tal que

$$
\|f(\sigma(t), x, u)\| \leq \gamma\|x\|+c
$$

para todo $(t, x, u) \in \mathbb{T} \times \mathbb{R}^{n} \times U(t)$.

Se o problema $(Q)$ admite um processo factivel $(x, u)$ então existe um processo ótimo $(\bar{x}, \bar{u})$.

Prova: Seja $F: \mathbb{T} \times \mathbb{R}^{n} \rightsquigarrow \mathbb{R}^{n}$ definida como

$$
F(t, x)=\{f(\sigma(t), x, u): u \in U(t)\} .
$$

Pela hipótese (ii), $F$ é convexa. Já das hipóteses (i) e (iii), $F$ é compacta.

Pela hipótese $(i)$ podemos concluir que a multifunção $F(t,):. \mathbb{R}^{n} \rightsquigarrow \mathbb{R}^{n}$ possui o gráfico fechado.

A função $h: \mathbb{T} \times \mathbb{R}^{n} \times \mathbb{R}^{m} \rightarrow \mathbb{R}^{n}$ dada por

$$
h(t, x, u)=f(\sigma(t), x, u)
$$

é uma $\Delta$-função de Carathéodory. Então a multifunção

$$
\begin{aligned}
H(t, x) & =h(t, x, U(t)) \\
& =f(\sigma(t), x, U(t))=F(t, x)
\end{aligned}
$$

será $\Delta \times \mathcal{B}^{n}$-mensurável.

Assim, usando o Teorema 12 e o Teorema 13 prova-se a existência de um processo ótimo $(\bar{x}, \bar{u})$ para o problema $(Q)$.

\section{Conclusões}

Esse artigo contribuiu para o avanço do conhecimento na teoria de escalas temporais. Mais especificamente, usando uma extensão do teorema de seleção de Filippov prova a existência de soluções para problemas de controle ótimo em escalas temporais. O resultado de seleção de Filippov foi discutido na Seção 4 e também é uma contribuição desse artigo.

\section{Referências}

Agarwal, R., Bohner, M., O'Regan, D. and Peterson, A. (2002). Dynamic equations on time scales: a survey, Journal of Computational and Applied Mathematics 141(1): 1-26. DOI: 10.1016/S0377-0427(01)00432-0

Bartle, R. G. (1995). The elements of integration and Lebesgue measure, John Wiley \& Sons Inc., New York. DOI: 10.1002/9781118164471

Bohner, M. and Guseinov, G. S. (2010). Surface areas and surface integrals on time scales, Dynam. Systems Appl. 19(3-4): 435-453. DOI: $10.1080 / 10652469.2010 .548335$

Bohner, M., Guseinov, G. S. and Karpuz, B. (2011). Properties of the Laplace transform on time scales with arbitrary graininess, Integral Transforms Spec. Funct. 22(11): 785800.

Bohner, M. and Peterson, A. (2003). Advances in dynamic equations on time scales, Birkhäuser Boston Inc., Boston, MA.

Cabada, A. and Vivero, D. R. (2005). Criterions for absolute continuity on time scales, Journal of Difference Equations and Applications 11(11): 1013-1028.

Cabada, A. and Vivero, D. R. (2006). Expression of the lebesgue $\delta$-integral on time scales as a usual lebesgue integral; application to the calculus of $\delta$-antiderivatives, Mathematical and Computer Modelling 43(1): 194-207. DOI: $10.1016 /$ j.mcm.2005.09.028

Castaing, C. and Valadier, M. (1977). Convex analysis and measurable multifunctions, Lecture Notes in Mathematics, Vol. 580, Springer-Verlag, Berlin. DOI: 10.1007/BFb0087685

Federson, M., Mesquita, J. G. and Slavík, A. (2012). Measure functional differential equations and functional dynamic equations on time scales, Journal of Differential Equations 252(6): $\quad 3816-3847 . \quad$ DOI: 10.1016/j.jde.2011.11.005 
Filippov, A. F. (1988). Differential equations with discontinuous righthand sides, Vol. 18 of Mathematics and its Applications (Soviet Series), Kluwer Academic Publishers Group, Dordrecht. Translated from the Russian.

Gong, Y. and Xiang, X. (2009). A class of optimal control problems of systems governed by the first order linear dynamic equations on time scales, Journal of Industrial and Management Optimization 5(1): 1-10.

Guseinov, G. S. (2003). Integration on time scales, Journal of Mathematical Analysis and Appli-cations 285(1): 107-127. DOI: 10.1016/S0022-247X(03)00361-5

Hilger, S. (1990). Analysis on measure chains - a unified approach to continuous and discrete calculus, Results Math. 18(1-2): 18-56. DOI: 10.1007/BF03323153

Hilscher, R. and Zeidan, V. (2011). First order conditions for generalized variational pro-blems over time scales, Computers \& Mathe-matics with Applications 62(9): 3490-3503. DOI: 10.1016/j.camwa.2011.08.065

Liu, G., Xiang, X. and Peng, Y. (2011). Nonlinear integro-differential equations and optimal control problems on time scales, Computers $\&$ Mathematics with Applications 61(2): 155169. DOI: $10.1016 /$ j.camwa.2010.10.013

Loewen, P. D. (1993). Optimal Control via Nonsmooth Analysis, CRM Proceedings and Lecture Notes, Vol. 2, American Mathematical Society, Providence.

Pawłuszewicz, E. and Torres, D. F. (2010). Avoidance control on time scales, Journal of optimization theory and applications 145(3): 527-542.

Peng, Y., Xiang, X. and Jiang, Y. (2011). Nonlinear dynamic systems and optimal control problems on time scales, ESAIM: Control, Optimisation and Calculus of Variations 17(03): 654-681. DOI: 10.1051/cocv/2010022

Royden, H. L. (1988). Real analysis, third edn, Macmillan Publishing Company, New York.

Rudin, W. (1987). Real and complex analysis, third edn, McGraw-Hill Book Co., New York.

Santos, I. L. D. (2011). Existência de soluções de inclusões diferenciais em escalas temporais, Tese de Doutorado, IBILCE-Unesp.

Santos, I. L. D. and Silva, G. N. (2013). Absolute continuity and existence of solutions to dynamic inclusions in time scales, Mathematische Annalen 356: 373-399. DOI: 10.1007/s00208-012-0851-8

Vinter, R. B. (2000). Optimal Control, Birkhauser, Boston.
Zhan, Z. and Wei, W. (2009). On existence of optimal control governed by a class of the firstorder linear dynamic systems on time scales, Applied Mathematics and Computation 215(6): 2070-2081. DOI: 10.1016/j.amc.2009.08.009

Zhan, Z., Wei, W. and Xu, H. (2009). Hamiltonjacobi-bellman equations on time scales, Mathematical and Computer Modelling 49(910): 2019-2028. DOI: 10.1016/j.mcm.2008.12.008 